\title{
The impact of vaccination status on importation of COVID-19 among international travellers
}

\author{
Paul Ronksley ${ }^{1 *}$, Tayler Scory ${ }^{2}$, Robert Weaver ${ }^{2}$, Meaghan Lunney ${ }^{1}$, Rachel Rodin ${ }^{3}$, Marcello Tonelli²
}

\begin{abstract}
Governments worldwide are looking for ways to safely enable international travel while mitigating the spread of severe acute respiratory syndrome coronavirus 2 (SARS-CoV-2), and the associated coronavirus disease 2019 (COVID-19). However, few data describe the impact of vaccination on importation of COVID-19. We took advantage of the sequential introduction of two government policies in Canada to evaluate the real-world evidence of vaccine effectiveness among 30,361 international travellers arriving by air in Alberta, Canada. The proportion of COVID-19-positive results for travellers who were either vaccinated or partially vaccinated was $0.02 \%$ (95\% Cl: $0.00-0.10$ ) (i.e. one positive case among 5,817 travellers). In contrast, $1.42 \%$ (95\% Cl: 1.27-1.58) of unvaccinated travellers tested positive for SARS-CoV-2 (341 cases among 24,034 travellers). These findings suggest that COVID-19 vaccinations approved in Canada, substantially reduced the risk of travel-related importation of COVID-19 when combined with other public health measures. The low absolute rate of infection among vaccinated or partially vaccinated international travellers may inform quarantine requirements in this population.
\end{abstract}

This work is licensed under a Creative Commons Attribution 4.0 International License.
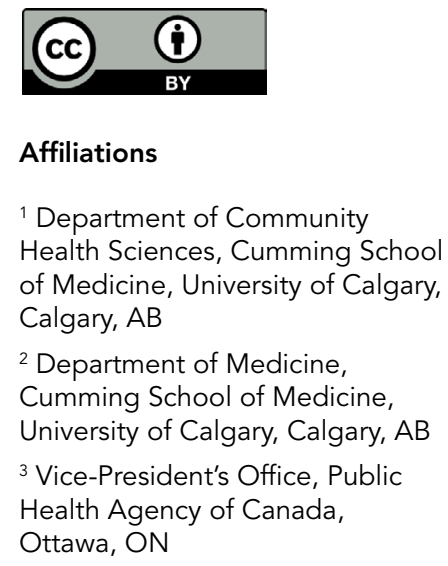

2 Department of Medicine, Cumming School of Medicine, University of Calgary, Calgary, AB

${ }^{3}$ Vice-President's Office, Public Health Agency of Canada, Ottawa, ON

\section{*Correspondence: \\ peronks|@ucalgary.ca}

Suggested citation: Ronksley PE, Scory TD, Weaver RG, Lunney M, Rodin R, Tonelli M. The impact of vaccination status on importation of COVID-19 among international travellers. Can Commun Dis Rep 2021;47(11):473-5.

https://doi.org/10.14745/ccdr.v47i11a05

Keywords: COVID-19, vaccination, international travel, case importation

\section{Introduction}

Governments worldwide are looking for ways to safely enable international travel while mitigating the spread of severe acute respiratory syndrome coronavirus 2 (SARS-CoV-2), and the associated coronavirus disease 2019 (COVID-19). Vaccination campaigns are well underway in many high-income countries, and accumulating evidence suggests that vaccinated people are less likely to become infected. Few data describe the impact of vaccination on importation of COVID-19, a potentially useful indicator to inform border policy. Using data from two government-sponsored border testing programs, we provide real-world evidence on vaccine effectiveness among 30,361 international travellers arriving by air at the Calgary International Airport.

\section{Current situation}

In March 2020, the Canadian federal government closed borders to everyone except people meeting certain criteria (1). Of those allowed to cross the border, one group was termed "exempt travellers", and included members of flight crews, those transporting goods across the Canada-United States border, and others providing certain essential services. The remainder of those allowed to cross the border were termed "non-exempt travellers", including Canadian citizens; permanent residents; the immediate families of citizens/permanent residents; and others with a specific reason for travel, such as family reunification. The Calgary International Airport was one of four airports that remained open to flights from outside of Canada, the United States and the Caribbean, along with airports in Montréal, Toronto and Vancouver.

Data from January 6, 2021 to February 21, 2021 were obtained from non-exempt travellers who were eligible to enter Canada, arrived by air, and participated in the voluntary Alberta Border Testing Pilot Program (ABTPP) (2). The ABTPP was suspended at 11:59 p.m. EST on February 21, 2021 and all international travellers arriving in Alberta by air thereafter were subject to a mandatory border entry procedure (3). We report here on travellers from both the ABTPP and the mandatory program from January 6, 2021 to May 23, 2021. These travellers are called thereafter the 'participants'.

All travellers in the current report were required to present proof of a negative molecular test for SARS-CoV-2 (e.g. Polymerase chain reaction (PCR), Nucleic acid test (NAT), Reverse transcription loop-mediated isothermal amplification (RT-LAMP), etc.), done fewer than 72 hours before the scheduled 
departure to Canada (4), and to undergo molecular testing again 7-8 days after arrival. Full-vaccination status was defined by self-reported receipt of two doses of a COVID-19 vaccine approved in Canada (5) at least 14 days prior to arrival date. Partial vaccination was defined by self-reported receipt of one Canadian-approved COVID-19 vaccine dose prior to arrival date or two Canadian-approved COVID-19 vaccine doses less than 14 days prior to arrival date. Recipients of a single-dose vaccine were considered partially vaccinated. Those with no self-reported vaccination status were linked, where possible, to the provincial vaccination registry to determine their status at time of arrival date. Most of the international travellers were Alberta residents returning from abroad and provided a provincial healthcare number.

Of the 30,361 non-exempt travellers, the majority traveled on flights originating in the United States (53.20\%) and Mexico (20.79\%). Their median age was 45.0 years and $52.5 \%$ were male. 28,658 (94.39\%) were at least 12 years of age, of whom 1,595 (5.57\%) were partially vaccinated and 4,227 (14.75\%) were fully vaccinated. The proportion of positive results for participants who were either fully vaccinated or partially vaccinated was $0.02 \%$ ( $95 \% \mathrm{Cl}: 0.00-0.10$ [i.e. one positive case among 5,817 participants who were tested for COVID-19]) (Table 1). In contrast, $1.42 \%$ (95\% Cl: $1.27-1.58)$ of unvaccinated participants tested positive for SARS-CoV-2 (341 cases among 24,034 participants). This equates to a relative risk for a positive test among vaccinated or partially vaccinated participants of 0.01 (95\% Cl: 0.00-0.09) compared with participants who were unvaccinated.

The positive test among the vaccinated traveller was followed up with sequencing and was negative for a variant of concern. Another specimen was obtained from this traveller three days later and a repeat molecular test was negative for SARS-CoV-2which raised the possibility of an initial false positive test.

\section{Conclusion}

These findings suggest that Canadian-approved COVID-19 vaccinations substantially reduce the risk of travel-related importation of COVID-19 when combined with other public health measures. The low absolute rate of infection among vaccinated or partially vaccinated non-exempt travellers may inform quarantine requirements in this population. However, recognizing the timeframe of this report, future work should investigate whether the effectiveness of vaccinations in mitigating case importation among international travellers has changed following the recent increase in variants of concern (particularly the Delta variant).

\section{Authors' statement}

PER - Contributions to conception and design, interpreted of data; wrote first draft

RGW - Contributions to conception and design, interpreted of data

$\mathrm{ML}$ - Contributions to conception and design, interpreted of data

RR - Contributions to conception and design, interpreted of data

MT - Contributions to conception and design, interpreted of data, wrote first draft

TDS - Data analysis

All authors were involved in revising it critically for important intellectual content. All authors provide final approval of the version to be published and agreed to be accountable for all aspects of the work.

\section{Competing interests}

None.

\section{Funding}

This work was funded by a contract from the Government of Alberta, which received a grant from the Public Health Agency of Canada.

Table 1: COVID-19 test results among non-exempt international travellers arriving in Alberta by air between January 6, 2021 and May 23, 2021

\begin{tabular}{|c|c|c|c|c|c|c|c|c|c|c|c|c|c|c|c|}
\hline \multirow{2}{*}{$\begin{array}{c}\text { Test } \\
\text { results }\end{array}$} & \multicolumn{3}{|c|}{ Vaccinated } & \multicolumn{3}{|c|}{ Partially vaccinated } & \multicolumn{3}{|c|}{ Unvaccinated } & \multicolumn{3}{|c|}{ Missing/unknown } & \multicolumn{3}{|c|}{ Total } \\
\hline & $\mathbf{n}$ & $\%$ & $95 \% \mathrm{Cl}$ & $\mathbf{n}$ & $\%$ & $95 \% \mathrm{Cl}$ & $\mathbf{n}$ & $\%$ & $95 \% \mathrm{Cl}$ & $\mathbf{n}$ & $\%$ & $95 \% \mathrm{Cl}$ & $\mathbf{n}$ & $\%$ & $95 \% \mathrm{Cl}$ \\
\hline Positive $^{a}$ & 1 & 0.02 & $0.00-0.13$ & 0 & 0.00 & $0.00-0.23$ & 341 & 1.42 & $1.27-1.58$ & 0 & 0.00 & $0.00-7.11$ & 342 & 1.14 & $1.03-1.27$ \\
\hline Negative & 4,224 & 99.98 & - & 1,592 & 100 & - & 23,693 & 98.58 & - & 50 & 100 & - & 29,559 & 98.85 & - \\
\hline No test & 2 & - & - & 3 & - & - & 455 & - & - & 0 & - & - & 460 & - & - \\
\hline Total & 4,227 & 13.92 & - & 1,595 & 5.25 & - & 24,489 & 80.66 & - & 50 & 0.16 & - & 30,361 & 100 & - \\
\hline
\end{tabular}

Abbreviations: $\mathrm{Cl}$, confidence interval;,- not reported

a Proportion of positive results for participants who were either vaccinated or partially vaccinated was $0.02 \%(0.00 \%, 0.10 \%)$ 


\section{References}

1. Public Health Agency of Canada. List of Acts and Regulations. Ottawa (ON): PHAC; 2021 (accessed 2021-06-07). https://www.canada.ca/en/public-health/ corporate/mandate/about-agency/acts-regulations/list-actsregulations.html

2. Government of Canada. Alberta COVID-19 Border Testing Pilot Program. Ottawa (ON): Government of Canada; 2021 (accessed 2021-06-07). https://www.canada.ca/en/publichealth/services/diseases/2019-novel-coronavirus-infection/ latest-travel-health-advice/alberta-covid-19-border-testingpilot-program.html

3. Government of Canada. Government of Canada introduces further restrictions on international travel. Ottawa (ON): Government of Canada; 2021 (accessed 2021-06-07). https://www.canada.ca/en/transport-canada/news/2021/01/ government-of-canada-introduces-further-restrictions-oninternational-travel.html
4. Government of Canada. COVID-19 testing for travellers. Ottawa (ON): Government of Canada; 2021 (accessed 2021-06-07). https://travel.gc.ca/travel-covid/travelrestrictions/flying-canada-checklist/covid-19-testingtravellers-coming-into-canada

5. Government of Canada. Approved COVID-19 Vaccines. Ottawa (ON): Government of Canada; 2021 (accessed 2021-06-07). https://www.canada.ca/en/health-canada/ services/drugs-health-products/covid19-industry/drugsvaccines-treatments/vaccines.html 